\title{
Cosmological Dynamics of a Hybrid Chameleon Scenario
}

\author{
Kourosh Nozari ${ }^{1}$ and Narges Rashidi ${ }^{2}$ \\ ${ }^{1}$ Research Institute for Astronomy and Astrophysics of Maragha (RIAAM), P.O. Box 55134-441, Maragha, Iran \\ ${ }^{2}$ Department of Physics, Faculty of Basic Sciences, University of Mazandaran, P.O. Box 47416-95447, Babolsar, Iran
}

Correspondence should be addressed to Kourosh Nozari; knozari@umz.ac.ir

Received 8 December 2012; Accepted 12 February 2013

Academic Editor: Joseph Formaggio

Copyright (C) 2013 K. Nozari and N. Rashidi. This is an open access article distributed under the Creative Commons Attribution License, which permits unrestricted use, distribution, and reproduction in any medium, provided the original work is properly cited.

\begin{abstract}
We consider a hybrid scalar field which is nonminimally coupled to the matter and models a chameleon cosmology. By introducing an effective potential, we study the dependence of the effective potential's minimum and hybrid chameleon field's masses on the local matter density. In a dynamical system technique, we analyze the phase space of this two-field chameleon model, find its fixed points and study their stability. We show that the hybrid chameleon domination solution is a stable attractor and the universe in this setup experiences a phantom divide crossing.
\end{abstract}

\section{Introduction}

Recent cosmological observations have revealed that our universe is currently undergoing an accelerating phase of expansion [1-14]. This transition to the accelerating phase has occurred in the recent cosmological past. Several approaches have been proposed to explain this late-time acceleration of the universe. Introducing some sort of unknown energy component (dubbed "dark energy"), with negative pressure, is one of these various approaches [15-37]. The simplest candidate for dark energy is the cosmological constant. However, the cosmological constant suffers from serious problems such as huge amount of fine tuning required for its magnitude and other theoretical problems such as unknown origin and lake of dynamics [38-41]. Nevertheless, the standard cosmological model with a cosmological constant has no internal problems or inconsistencies both at the classical and quantum levels [42].

In this regard, the scalar fields, such as quintessence [43, 44], phantom fields [45], and tachyon fields [46, 47] and so on, provide a simple dynamical model for dark energy which can explain cosmic accelerating expansion. The scalar fields can directly couple to matter Lagrangian or indirectly couple to the Ricci scalar [48-51]. If there are negligible selfinteractions for scalar fields, then the experimental bounds on such fields require them either to couple to the matter much more weakly than the gravity does, or to couple very strongly [52-55]. Such fields must be very light (their mass must be of the order of $H_{0}$, the present Hubble parameter) in order to evolve cosmologically today. Also, in order to have consistency with Equivalence Principal, their coupling to the matter must be extremely small. Chameleon cosmology $[56,57]$ is a scenario which can address this problem suitably. In the chameleon cosmology, the scalar fields evolve on the cosmological time scales today, while according to the expectations from string theory they have couplings of order unity to the matter and at the same time remain very light on the cosmological scales. In fact, the mass of the scalar field is not constant and, instead, it depends on the local matter density. In the high density regime, the mass of the scalar fields is large, so that the resulting violations of the Equivalence Principal are exponentially suppressed. On the cosmological scales, where the density is very low, the mass of the fields can be of the order of the present Hubble parameter $\left(H_{0}\right)$ and cause the current acceleration of the universe. Such a scalar field is dubbed "chameleon" because its physical properties, such as its mass, depend on its environment [56, 57]. We note that this definition of the "chameleon" field is too wide and includes situations such as plasma density dependence of plasmon mass and also density and temperature dependence of elementary particles masses through their effective potentials. In our setup the term chameleon 
has sense only in the case of specific exponential coupling of known quantum fields of matter to the chameleon field [53].

The present work has been organized as follows: In Section 2, we introduce the idea of chameleon cosmology. We consider a quintom scalar field which is nonminimally coupled to the matter (a quintom field is a hybrid of a quintessence and a phantom field [58-63]). In this section, by means of the conservation equation, we derive the matter energy density which depends on the hybrid scalar field. We study the effective chameleon potential, its minimum, and the mass of two fields about the minimum of the potential. We show that the value of the scalar fields and their masses at the minimum depend on local energy density. In Section 3, we study the cosmological dynamics of the model in the dynamical system approach and we provide a detailed phase space analysis of the model. We find that in the parameters space of the model it is possible to have a chameleon dominated stable attractor which has a negative effective equation of state. We also show that, in this setup, the effective equation of state parameter of the model crosses the phantom divide line and the deceleration parameter becomes negative in the past. So this model has the capability to explain the late-time cosmic speedup.

\section{The Setup}

The action of a two-field chameleon model in 4 dimensions can be written as follows:

$$
\begin{gathered}
S=\int d^{4} x \sqrt{-g}\left(\frac{R}{2 \kappa^{2}}+\frac{1}{2} g^{\mu \nu} \partial_{\mu} \phi \partial_{\nu} \phi-\frac{1}{2} g^{\mu \nu} \partial_{\mu} \varphi \partial_{\nu} \varphi\right. \\
\left.+V(\phi, \varphi)+\mathscr{L}_{m}\left(\psi^{(i)}, g_{\mu \nu}^{(i)}\right)\right),
\end{gathered}
$$

where $\kappa^{2}=8 \pi / M^{2}$ is the gravitational coupling and $\mathscr{L}_{m}$ represents the Lagrangian density of the matter fields. Also, the matter fields $\psi^{(i)}$ are coupled to the scalar fields by the definition $g_{\mu \nu}^{(i)}=e^{2 \kappa \beta_{(i)}(\phi+\varphi)} g_{\mu \nu}$, where $\beta_{(i)}$ are dimensionless constants. In this paper, we assume just a single matter energy density component $\left(\rho_{m}\right)$ with coupling $\beta$. Also, according to the expectations from string theory, we allow $\beta$ to be of the order of unity.

In a flat Friedmann-Robertson-Walker background, variation of action (1) with respect to the metric leads to the following equations:

$$
\begin{gathered}
H^{2}=\frac{\kappa^{2}}{6} \dot{\phi}^{2}-\frac{\kappa^{2}}{6} \dot{\varphi}^{2}+\frac{\kappa^{2}}{3} V(\phi, \varphi)+\frac{\kappa^{2}}{3} \rho_{m} e^{\kappa \beta(\phi+\varphi)}, \\
2 \dot{H}+3 H^{2}=-\frac{\kappa^{2}}{2} \dot{\phi}^{2}+\frac{\kappa^{2}}{2} \dot{\varphi}^{2}+\kappa^{2} V(\phi, \varphi)-\kappa^{2} \omega \rho_{m} e^{\kappa \beta(\phi+\varphi)} .
\end{gathered}
$$

To derive these equations we have assumed that the matter field of the universe is a perfect fluid, so that $p_{m}=\omega \rho_{m}$. Variation of the action (1) with respect to the scalar fields gives the following equation of motion:

$$
\ddot{\phi}-\ddot{\varphi}+3 H(\dot{\phi}-\dot{\varphi})+\frac{d V}{d \phi}+\frac{d V}{d \varphi}-2 \kappa \beta \omega \rho_{m} e^{\kappa \beta(\phi+\varphi)}=0 .
$$

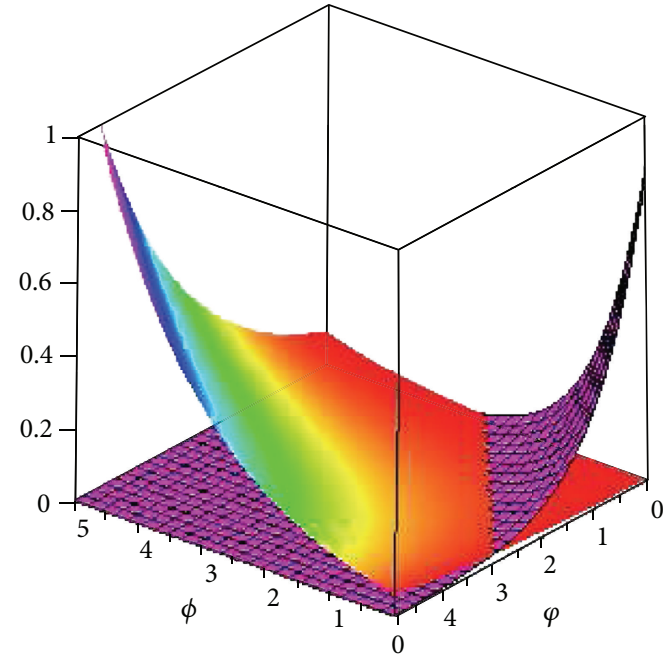

FIGURE 1: The behavior of the runaway potential (chromatic surface) and the coupling term $e^{\kappa \beta(\phi+\varphi)}$ (the magenta meshed surface) with respect to the two components $(\phi$ and $\varphi$ ) of the hybrid scalar field. By increment of the scalar fields, the runaway potential decreases, while the coupling term increases.

Equations (2)-(4) give us the energy conservation equation of the model as follows:

$$
\dot{\rho}_{m}+3 H \rho_{m}(1+\omega)=-\kappa \beta(1+\omega) \rho_{m}(\dot{\phi}+\dot{\varphi}) .
$$

The right hand side of (5) shows the nonconservation of the energy density in this setup, which is due to the presence of nonminimal coupling between the hybrid scalar field and matter Lagrangian (in other words, it is due to the presence of the chameleon field). In the absence of chameleon field (the case that $\beta$ goes to zero), (5) simplifies to the ordinary conservation equation.

If we integrate (5), we reach the following expression for the matter energy density:

$$
\rho_{m}=C a^{-3(1+\omega)} e^{\kappa \beta(\phi+\varphi)},
$$

where $C$ is a constant. This equation shows that the scalar fields and the matter energy density are related to each other via the chameleon coupling term.

By using (2) and (3), we can deduce the effective equation of state parameter in our setup as follows:

$$
\omega_{\text {eff }}=\frac{p_{\text {eff }}}{\rho_{\text {eff }}}=\frac{\dot{\phi}^{2}-\dot{\varphi}^{2}-2 V(\phi, \varphi)+2 \omega \rho_{m} e^{\kappa \beta(\phi+\varphi)}}{\dot{\phi}^{2}-\dot{\varphi}^{2}+2 V(\phi, \varphi)+2 \rho_{m} e^{\kappa \beta(\phi+\varphi)}} .
$$

Equation (4) shows that the dynamics of hybrid scalar field in this case do not just depend only on the $V(\phi, \varphi)$, but instead it depends on an effective potential which is defined as follows:

$$
V_{\text {eff }}(\phi, \varphi)=V(\phi, \varphi)+\kappa \beta \rho_{m} e^{\kappa \beta(\phi+\varphi)},
$$

which is usually dubbed chameleon effective potential. This effective potential depends explicitly on the matter density $\rho_{m}$. We note that we assume a runaway potential defined as

$$
V(\phi, \varphi)=V_{0} e^{-\sqrt{6} \kappa(m \phi+n \varphi)},
$$


which decreases by increment of the scalar fields. Also, the coupling term $\left(e^{\kappa \beta(\phi+\varphi)}\right)$ increases as the scalar fields increase (see Figure 1).

So, if $\beta>0, V_{\text {eff }}$ has minimum. The behavior of the effective potential with respect to $\phi$ and $\varphi$ is shown in Figure 2 .
We denote the values of $\phi$ and $\varphi$, where the effective potential becomes minimum (the derivative of the effective potential becomes zero), by $\phi_{\min }$ and $\varphi_{\min }$ respectively. Then, the minimum of the effective potential in our model occurs at

$$
\left(\phi_{\min }, \varphi_{\min }\right)=\left(\phi_{*}, \frac{-\ln \left(\beta^{2} \kappa^{2} C(-1-\omega+m) / \sqrt{6} m a^{3(1+\omega)}\right)+\beta \kappa \phi_{*}(1+\omega)-\beta \kappa n \phi_{*}-\sqrt{6} n \phi_{*}}{\beta \kappa m-\beta \kappa \omega-\beta \kappa+\sqrt{6} m}\right) .
$$

The above expression shows that the minimum of the effective chameleon potential is a line. Since we deal with positive scalar field, $\varphi_{\min }$ leads us to a constraint on $\phi$ as follows:

$$
\phi_{*} \geq \frac{\ln \left(\beta^{4} \kappa^{4} C^{2}(-1-\omega-m)^{2} / 6 m^{2} a^{6(1+\omega)}\right)}{2 \beta \kappa(1+\omega)-2 \beta \kappa n-2 \sqrt{6} n} .
$$

This means that if $\phi$ satisfies the constraint equation (11), there is a minimum for effective potential as is shown in Figure 2.

The mass of the scalar field $\phi$ about the minimum is obtained from the following relation:

$$
m_{\phi}^{2}=\left.\frac{\partial^{2} V_{\mathrm{eff}}}{\partial \phi^{2}}\right|_{\phi=\phi_{\min }}
$$

So, we find

$$
\begin{aligned}
\left(m_{\phi}\right)_{\min }^{2}= & 6 m^{2} V_{\min }+\kappa^{2} \beta^{2} \rho_{m} e^{\kappa \beta\left(\phi_{\min }+\varphi_{\min }\right)} \\
& +\kappa^{2} \beta^{2}\left(1+\omega^{2}\right) \rho_{m} e^{\kappa \beta\left(\phi_{\min }+\varphi_{\min }\right)}
\end{aligned}
$$

Also the mass of the scalar field $\varphi$ is given by

$$
m_{\varphi}^{2}=\left.\frac{\partial^{2} V_{\mathrm{eff}}}{\partial \varphi^{2}}\right|_{\varphi=\varphi_{\min }}
$$

leading to the result

$$
\begin{aligned}
\left(m_{\varphi}\right)_{\min }^{2}= & 6 n^{2} V_{\min }+\kappa^{2} \beta^{2} \rho_{m} e^{\kappa \beta\left(\phi_{\min }+\varphi_{\min }\right)} \\
& +\kappa^{2} \beta^{2}\left(1+\omega^{2}\right) \rho_{m} e^{\kappa \beta\left(\phi_{\min }+\varphi_{\min }\right)} .
\end{aligned}
$$

As (13) and (15) show, $\left(m_{\phi}\right)_{\min }$ and $\left(m_{\varphi}\right)_{\min }$, are increasing functions of local matter density, $\rho_{m}$. This means that the larger values of matter density lead to larger values of the chameleon field's mass.

\section{Cosmological Dynamics}

In this section, we are going to study cosmological dynamics of the hybrid chameleon model introduced in the previous section in the framework of dynamical system analysis and phase space trajectories of the model. In this regard, we should firstly introduce some new convenient dimensionless variables. These dimensionless quantities help us to translate our equations of the cosmological dynamics in the language of the autonomous dynamical system. In our setup, the dimensionless parameters are defined as follows:

$$
\begin{gathered}
x=\frac{\kappa \dot{\phi}}{\sqrt{6} H}, \quad y=\frac{\kappa \dot{\varphi}}{\sqrt{6} H}, \quad z=\frac{\kappa \sqrt{V}}{\sqrt{3} H}, \\
u=\frac{\kappa \sqrt{\rho_{m} e^{\kappa \beta(\phi+\varphi)}}}{\sqrt{3} H} .
\end{gathered}
$$

By rewriting the Friedmann equation (2) in terms of the new variables, we reach a constraint on the parameters space of the model as follows:

$$
1=x^{2}-y^{2}+z^{2}+u^{2}
$$

by which we can express one of the dimensionless variables in terms of the others.

By introducing a new time variable $\tau \equiv \ln a$, we obtain the following autonomous system in our setup:

$$
\begin{aligned}
\frac{d x}{d \tau}= & -3 x+3 m z^{2}+\frac{\sqrt{6}}{2} \beta \omega u^{2} \\
& +\frac{3 x}{2}\left[1+x^{2}-y^{2}-z^{2}+\omega u^{2}\right], \\
\frac{d y}{d \tau}= & -3 y-3 n z^{2}-\frac{\sqrt{6}}{2} \beta \omega u^{2} \\
& +\frac{3 x}{2}\left[1+x^{2}-y^{2}-z^{2}+\omega u^{2}\right], \\
\frac{d z}{d \tau}=-3 m z x & -3 n z y+\frac{3 z}{2}\left[1+x^{2}-y^{2}-z^{2}+\omega u^{2}\right], \\
\frac{d u}{d \tau}=- & \frac{3}{2}(1+\omega) u-\frac{\beta}{2} \omega(\sqrt{6} x+\sqrt{6} y) u \\
+ & \frac{3 u}{2}\left[1+x^{2}-y^{2}-z^{2}+\omega u^{2}\right] .
\end{aligned}
$$

Now, in order to analyze the cosmological evolution in the dynamical system approach, we should find fixed (or critical) points of the model. Fixed points are defined as those points that all autonomous equations (18) vanish. Eliminating $z$ by using constraint equation (17), we obtain the critical points of our setup. We find four critical points $(\mathbf{M}, \mathbf{N}, \mathbf{P}$, and $\mathbf{Q})$ and two critical lines $\left(\mathscr{L}_{1}\right.$ and $\left.\mathscr{L}_{2}\right)$ in our setup which we have summarized their properties in Tables 1,2 , and 3.

Now we discuss characters of each critical point separately. 


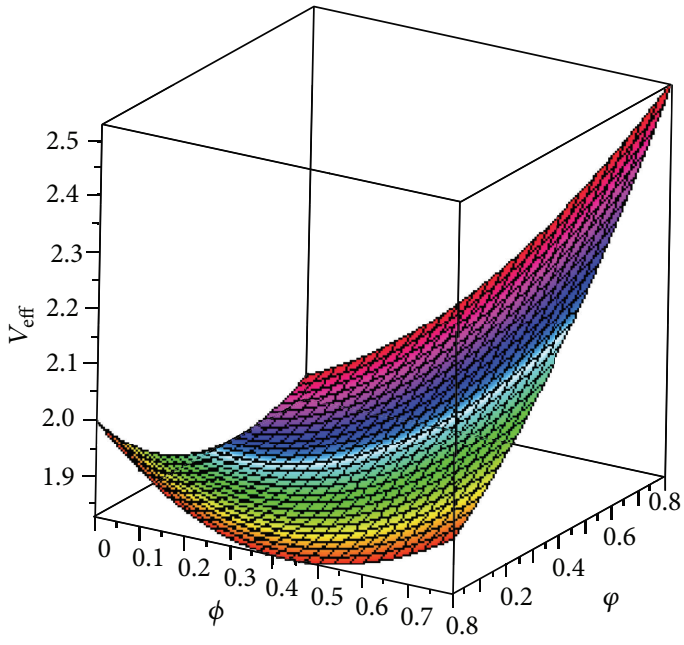

FIGURE 2: The behavior of the effective chameleon potential with respect to $\phi$ and $\varphi$. The effective chameleon potential reaches a minimum (a line in this case) during its evolution.

TABLE 1: Location and existence of critical points and corresponding effective equation of state parameter. $m_{*}$ and $\left(x_{\mathrm{Q}}, y_{\mathrm{Q}}, u_{\mathrm{Q}}\right)$ are defined by (19) and (20), respectively.

\begin{tabular}{lccc}
\hline Point & $(x, y, u)$ & Existence & $\omega_{\text {eff }}$ \\
\hline $\mathbf{M}$ & $(m,-n, 0)$ & $\begin{array}{c}\text { All } m \text { and } n \\
\text { and all } \omega\end{array}$ & $2 m^{2}-2 n^{2}-1$ \\
$\mathbf{N}$ & $\left(\frac{\sqrt{6} \beta \omega}{3-3 \omega}, \frac{\sqrt{6} \beta \omega}{3 \omega-3}, 1\right)$ & $\begin{array}{c}\text { All } m \text { and } n \\
\text { and } \omega \neq 1\end{array}$ & $\omega$ \\
$\mathbf{P}$ & $\left(\frac{\sqrt{6} \beta \omega}{3-3 \omega}, \frac{\sqrt{6} \beta \omega}{3 \omega-3},-1\right)$ & $\begin{array}{c}\text { All } m \text { and } n \\
\text { and } \omega \neq 1\end{array}$ & $\omega$ \\
$\mathbf{Q}$ & $\left(x_{\mathrm{Q}}, y_{\mathrm{Q}}, u_{\mathrm{Q}}\right)$ & $\begin{array}{c}m \geq m_{*} \text { and all } \\
\omega\end{array}$ & $\frac{x_{\mathrm{Q}}^{2}-y_{\mathrm{Q}}^{2}-z_{\mathrm{Q}}^{2}+\omega u_{\mathrm{Q}}^{2}}{x_{\mathrm{Q}}^{2}-y_{\mathrm{Q}}^{2}+z_{\mathrm{Q}}^{2}+u_{\mathrm{Q}}^{2}}$ \\
\hline
\end{tabular}

Critical Point M. Point $\mathbf{M}$ represents either a solution with a scalar field's kinetic energy term domination or potential energy term domination, depending on the values of $m$ and $n$. Also, depending on these values, this solution can be stable or unstable. For instance, by taking $m=0.6$ and $n=0.5$, the universe with this solution is an attractor, potential energy term dominated, meaning that if the universe reaches this state, it remains there forever. The value of effective equation of state parameter corresponding to this solution is negative $(-0.78)$, so this case is corresponding to an accelerating universe. Figure 3 shows the phase space trajectories of the model in two dimensions for $\beta=1, m=0.6, n=0.5$, and $\omega=0$ (corresponding to potential energy domination). The point $\mathbf{M}$ is shown as an attractor point in this plot. Also, we can see this stable point in 3-dimensional plot as shown in Figure 4. If we set $n=0.7$ and $m=1.2$, the solution is a saddle, kinetic energy domination. The universe during its evolution can reach this state but does not remain there and evolves to another state. In this case, the corresponding value of the effective equation of state parameter is positive $\left(\omega_{\text {eff }}=0.9\right)$ and shows a decelerating universe. This saddle point of the phase space trajectories is shown in Figure 5. This figure is plotted for $n=0.7$ and $m=1.2$. The point $\mathbf{M}$ would be corresponding to a repeller, kinetic term dominated solution if for instance we set $m=1.6, n=0.8$, and $\omega=1 / 3$ (see Figure 6). This unstable solution is relevant to early times cosmology.

Critical Point N. Critical point $\mathbf{N}$ exists if $\omega \neq 1$ (in other words, if there is no stiff fluid in the universe). For this solution, the effective equation of state parameter is exactly the same as the equation of state parameter of the matter and so the universe with this solution is effectively matter dominated. For $\omega<1$, depending on the values of $m$ and $n$, this matter dominated solution can be stable or saddle point. Also, with $\omega=0$, this solution is always a saddle, independent of the values of $m$ and $n$. This means that the universe, during its evolution, experiences this matter domination state and then evolves to another state. The point $\mathbf{N}$, in both Figures 4 and 5 , is a saddle point in the phase space of the model. It should be noticed that since both figures have been plotted with $\omega=0$, for both $m=0.6$ and $m=1.1, \mathbf{N}$ is a saddle point.

Critical Point $\mathbf{P}$. Like the critical point $\mathbf{N}$, the critical point $\mathbf{P}$ exists if $\omega \neq 1$. The properties of this critical point are the same as the properties of the critical point N. In Figure 4, we can see the point $\mathbf{P}$ as a saddle point in the phase space trajectories of the model. This point is not shown in Figure 3, because this figure has been plotted for positive $u$ while $\mathbf{P}$ is located at $u=-1$.

Critical Point $\mathbf{Q}$. The critical point $\mathbf{Q}$ exists if there is the following constraint on the parameters space of the model:

$$
m>\frac{\sqrt{6}}{12} \beta \omega+\frac{\sqrt{6 \beta^{2} \omega^{2}+72+144 n^{2}+72 \omega-24 \sqrt{6} \beta \omega n}}{12} \equiv m_{*} \text {. }
$$

If this critical point exists, its location is at

$$
\left(x_{\mathrm{Q}}, y_{\mathrm{Q}}, u_{\mathrm{Q}}\right) \text {, }
$$

where

$$
\begin{aligned}
& x_{Q}=\frac{12 n^{2} \beta \omega-3 m \sqrt{6}+6 \omega \beta-12 m n \beta \omega+3 m \sqrt{6} \omega^{2}+6 \omega^{2} \beta-2 \sqrt{6} \beta^{2} \omega^{2} n+2 \sqrt{6} \beta^{2} \omega^{2} m}{\sqrt{6}\left(-6 m \omega+6 m-6 n \omega+6 n+\sqrt{6} \omega^{2} \beta-3 \sqrt{6} \omega \beta\right)(n-m)}, \\
& y_{Q}=\frac{6 \beta \omega+6 \beta \omega^{2}-2 \sqrt{6} \beta^{2} \omega^{2} n-3 \sqrt{6} n+3 \sqrt{6} n \omega^{2}+12 m \beta \omega n+2 \sqrt{6} \beta^{2} \omega^{2} m-12 m^{2} \omega \beta}{\sqrt{6}\left(6 n+6 m-6 n \omega-6 m \omega-3 \beta \omega \sqrt{6}+\beta \omega^{2} \sqrt{6}\right)(m-n)},
\end{aligned}
$$




$$
\begin{aligned}
u_{Q}= & \frac{\sqrt{3 n+3 m-2 \beta \omega \sqrt{6}-6 n \omega-6 m \omega+2 \sqrt{6} \omega^{2} \beta+3 n \omega^{2}+3 \omega^{2} m-2 \beta^{2} \omega^{2} n+2 \beta^{2} \omega^{2} m}}{\left(-6 m \omega+6 m-6 n \omega+6 n+\sqrt{6} \omega^{2} \beta-3 \beta \omega \sqrt{6}\right)(n-m)^{1 / 2}} \\
& \times \sqrt{-12 m^{2}+6+12 n^{2}+6 \omega+2 \sqrt{6} m \omega \beta-2 \sqrt{6} \omega \beta n} .
\end{aligned}
$$

Also, $\left(\lambda_{1 Q}, \lambda_{2 Q}, \lambda_{3 Q}\right)$ are the eigenvalues of the following matrix

$$
M=\left(\begin{array}{lll}
\frac{\partial x^{\prime}}{\partial x} & \frac{\partial x^{\prime}}{\partial y} & \frac{\partial x^{\prime}}{\partial u} \\
\frac{\partial y^{\prime}}{\partial x} & \frac{\partial y^{\prime}}{\partial y} & \frac{\partial y^{\prime}}{\partial u} \\
\frac{\partial u^{\prime}}{\partial x} & \frac{\partial u^{\prime}}{\partial y} & \frac{\partial u^{\prime}}{\partial u}
\end{array}\right)_{(x, y, u)=\left(x_{Q}, y_{Q}, u_{Q}\right)}
$$

where prime refers to derivative with respect to $\tau$ (see (18)). We note that since the eigenvalues of this solution are so lengthy and complicated, we avoid to express them here explicitly. The universe with this solution experiences an accelerating phase if

$$
\begin{aligned}
& -\sqrt{y_{\mathrm{Q}}^{2}+\frac{z_{\mathrm{Q}}^{2}}{2}-u_{\mathrm{Q}}^{2}\left(\omega+\frac{1}{3}\right)} \\
& \leq x_{\mathrm{Q}} \leq \sqrt{y_{\mathrm{Q}}^{2}+\frac{z_{\mathrm{Q}}^{2}}{2}-u_{\mathrm{Q}}^{2}\left(\omega+\frac{1}{3}\right)} .
\end{aligned}
$$

Point $\mathbf{Q}$ of Figure 5 is a stable chameleon dominated solution and this means that, if the universe reaches this state, it remains there forever. In this solution, the universe can experience the late time acceleration. Figure 5 shows 3dimensional phase space of our setup with $\beta=1, \omega=0$, $m=1.2$, and $n=0.7$. With these values of the parameters, the critical points $\mathbf{M}$ and $\mathbf{N}$ are saddle points and the point $\mathbf{Q}$ is a stable attractor. In summary, we can say that, with these parameters values, the universe during its evolution reaches a kinetic energy dominated era and then evolves to a matter dominated era. After that the universe evolves to a chameleon dominated era and remains there forever. Also, with this choice of values, the value of the effective equation of state parameter is about -1.1 . This means that the universe in this parameters space experiences late time acceleration and its stable state lies in phantom-like phase.

Critical Line $\mathscr{L}_{1}$. In this model, we also have two critical lines. The critical line $\mathscr{L}_{1}$, which is located at $\left(x_{*}, \sqrt{x_{*}^{2}-1}, 0\right)$, is a quintessence's kinetic term dominated solution. So, there is a constraint on this solution. This kinetic energy dominated solution exists if

$$
x_{*} \geq 1 \quad \text { or } \quad x_{*} \leq 1
$$

The stability of this line depends on the model parameters values. In the parameters space which satisfies the following constraint, this solution is a stable attractor:

$$
\begin{aligned}
m>- & \frac{\sqrt{6} n \beta \omega x^{2}+\sqrt{6} x \sqrt{-1+x^{2}} n \beta \omega}{x\left(\beta \omega \sqrt{6} x+\beta \omega \sqrt{6} \sqrt{-1+x^{2}}+3 \omega-3\right)} \\
& -\frac{\beta \omega \sqrt{6} x-\sqrt{6} n \beta \omega-\beta \omega \sqrt{6} \sqrt{-1+x^{2}}}{x\left(\beta \omega \sqrt{6} x+\beta \omega \sqrt{6} \sqrt{-1+x^{2}}+3 \omega-3\right)} \\
& +\frac{+3+3 n \sqrt{-1+x^{2}} \omega-3 n \sqrt{-1+x^{2}}-3 \omega}{x\left(\beta \omega \sqrt{6} x+\beta \omega \sqrt{6} \sqrt{-1+x^{2}}+3 \omega-3\right)} \equiv m_{\mathscr{L}_{1}} .
\end{aligned}
$$

In Figure 3 where the chosen values of the parameters do not satisfy the constraint equation (25), the critical line $\mathscr{L}_{1}$ is unstable. But in Figure 6 this line is a stable attractor because the values of parameters used to plot this figure satisfy the mentioned constraint.

Critical Line $\mathscr{L}_{2}$. This phantom's kinetic energy dominated critical line is located at $\left(-x_{*},-\sqrt{x_{*}^{2}-1}, 0\right)$. So, for existence of this solution, the constraint equation (25) should be satisfied. In contrast to $\mathscr{L}_{1}$ case, the critical line $\mathscr{L}_{2}$ is an unstable solution for all values of the model parameters space (see Figures 3 and 6).

A parameter which gives us a suitable background to understand the dynamics of the universe, the nature of dark energy, and the possibility of crossing of the phantom-divide line is the equation of state parameter. According to the recent observational data, the equation of state parameter of the dark energy crossed the phantom divide line $(\omega=-1)$ in the near past. It is shown that considering a dynamical dark energy component in a cosmological setup enables the model to explain this observational evidence [15-37]. Figure 7 shows the evolution of $\omega_{\text {eff }}$ with respect to the redshift parameter in our setup. This figure has been plotted by adopting the ansatz with $a=a_{0} e^{\beta t}, \varphi=\varphi_{0} e^{-\delta t}$, and $\phi=\phi_{0} e^{-\alpha t}(\alpha, \delta$, and $\beta$ are positive constants). Note that, in this figure, the values of parameters are the same as Figure 5 (where the chameleon dominated solution is a stable attractor). Also, we have set $a_{0}=1, \phi_{0}=0.8, \alpha=1, \delta=1.6$ and $\beta=1$. As figure shows, in the presence of the chameleon field, the universe enters the phantom phase in the near past at $z \simeq 0.26$. So, in this model, the universe experiences a smooth crossing of the phantom divide, $\omega_{\text {eff }}=-1$, line. 
TABLE 2: Eigenvalues and dynamical characters of the fixed points.

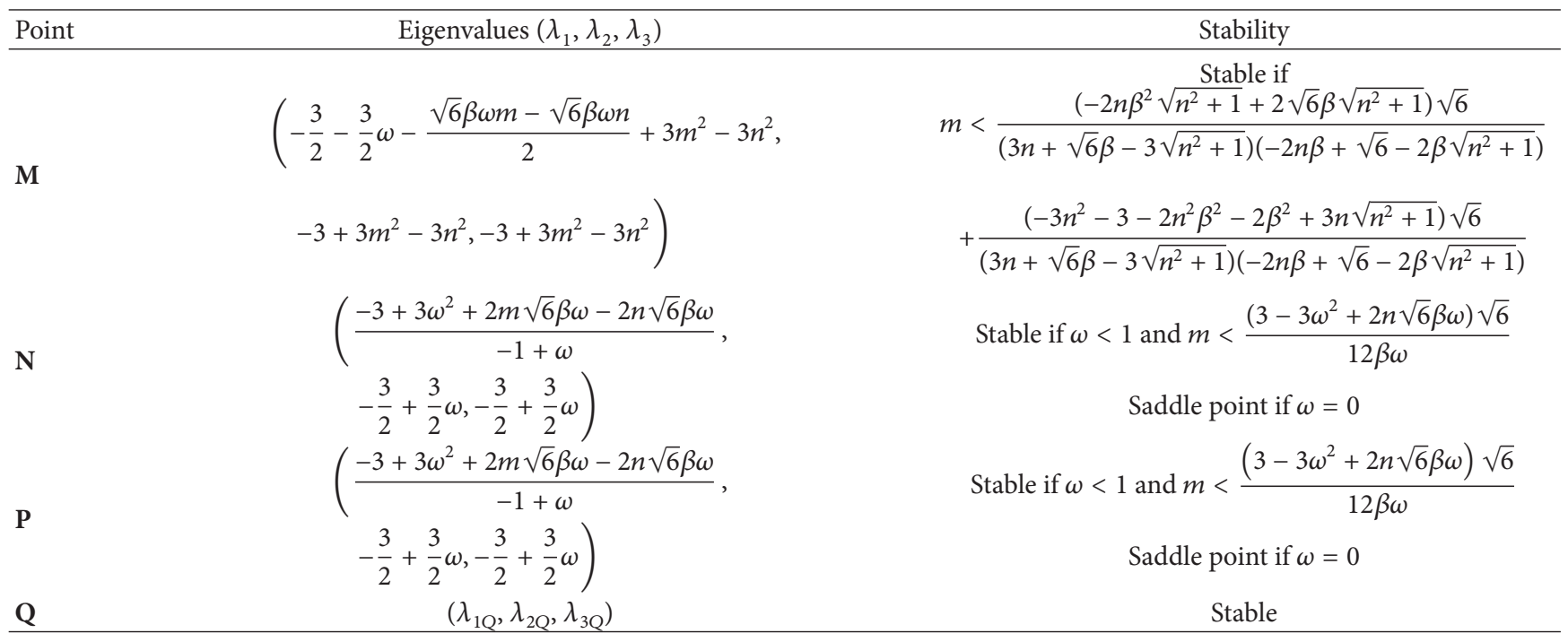

TABLE 3: Location, eigenvalues, and dynamical characters of the critical lines. Note that $m_{\mathscr{L}_{1}}$ is defined in (25).

\begin{tabular}{|c|c|c|c|c|c|}
\hline Line & $(x, y, u)$ & Existence & Eigenvalues & $\omega_{\text {eff }}$ & Stability \\
\hline $\mathscr{L}_{1}$ & $\left(x_{*}, \sqrt{x_{*}^{2}-1}, 0\right)$ & All $m, n$, and $\omega$ if $x \geq 1$ & $\left(\lambda_{1 \mathscr{L}_{1}}, \lambda_{2 \mathscr{L}_{1}}, \lambda_{3 \mathscr{L}_{1}}\right)$ & 1 & Stable if $m>m_{\mathscr{L}_{1}}$ \\
\hline $\mathscr{L}_{2}$ & $\left(x_{*},-\sqrt{x_{*}^{2}-1}, 0\right)$ & All $m, n$, and $\omega$ if $x \leq-1$ & $\left(\lambda_{1 \mathscr{L}_{2}}, \lambda_{2 \mathscr{L}_{2}}, \lambda_{3 \mathscr{L}_{2}}\right)$ & 1 & Unstable \\
\hline
\end{tabular}

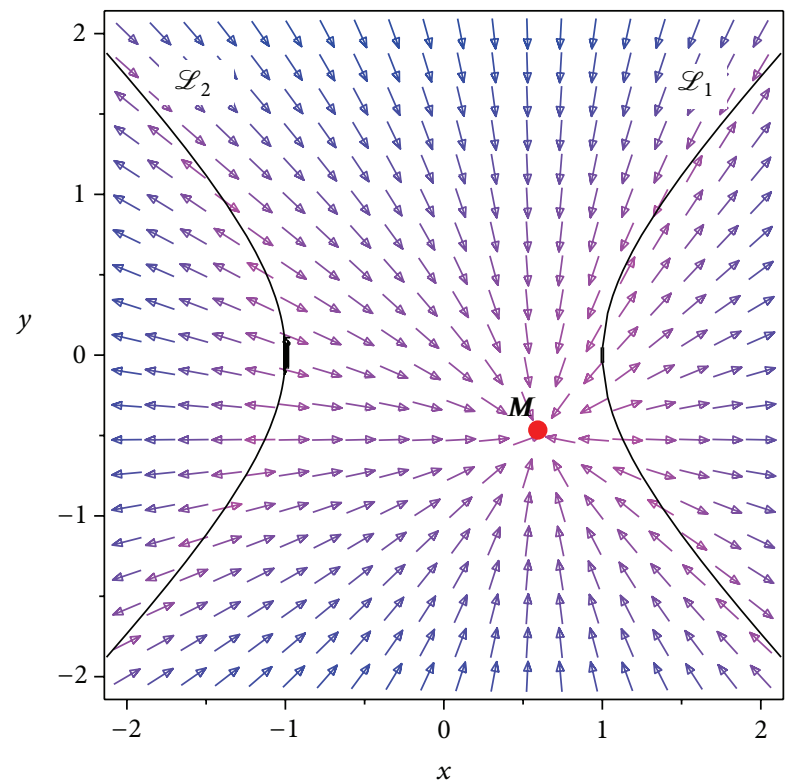

Figure 3: The phase space trajectories of the hybrid chameleon model with $m=0.6, n=0.5, \beta=1$, and $\omega=0$. This figure is plotted for the case with $u=0$. With these values in the parameters space, the critical lines $\mathscr{L}_{1}$ and $\mathscr{L}_{2}$ (the solutions corresponding to the quintessence component's kinetic energy domination) are repellers (and so unstable solutions). The critical point $\mathbf{M}$ (the solution with potential energy domination) is a stable attractor.

The deceleration parameter, $q$, is another important parameter in cosmological evolution. Its positive value (corresponding to $\ddot{a}<0$ ) shows the decelerating expansion of

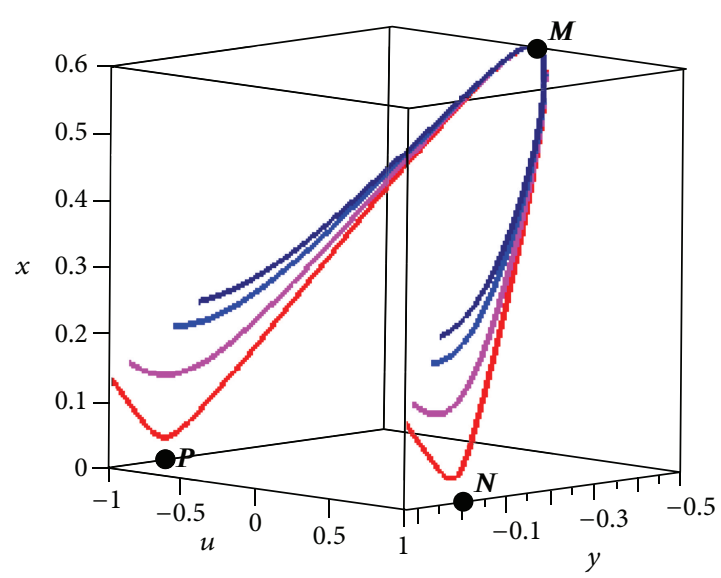

FIGURE 4: 3-dimensional phase space trajectories of the model with $m=0.6, n=0.5, \beta=1$, and $\omega=0$. For these values of parameters, we have a potential energy dominated solution (point $\mathbf{M}$ ) which is a stable attractor. The points $\mathbf{N}$ and $\mathbf{P}$ (corresponding to the effectively matter dominated era) are saddle points.

the universe and its negative value (corresponding to $\ddot{a}>0$ ) shows the accelerating expansion. $q$ is defined as follows:

$$
q=-\frac{\dot{H}}{H^{2}}-1
$$

The evolution of the deceleration parameter versus redshift is plotted in Figure 8. We can see from this figure that the deceleration parameter has become negative in the past at $z \simeq 0.84$, meaning that the universe has entered an accelerating phase at $z \simeq 0.84$. So a hybrid chameleon model 


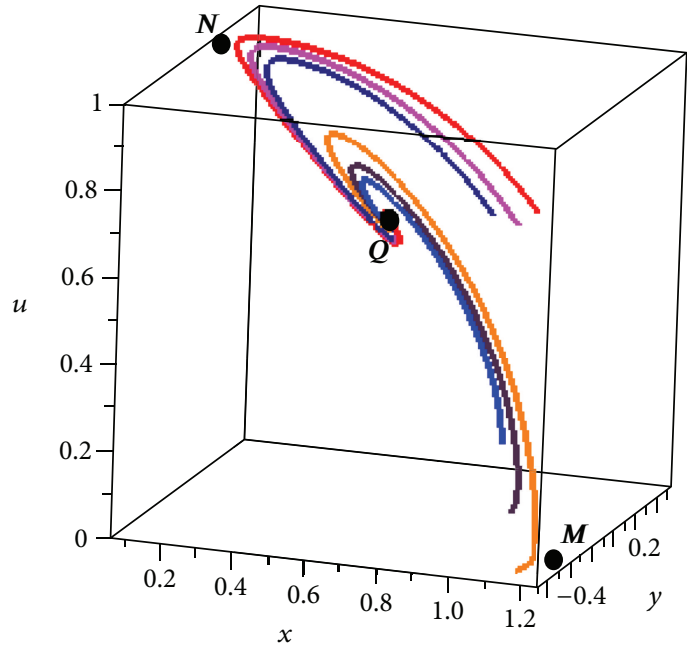

FIGURE 5: 3-dimensional phase space trajectories of the model with $m=1.2, n=0.7, \beta=1$ and $\omega=0$. For these values of parameters, the potential energy dominated solution (point $\mathbf{M}$ ) is a saddle point, while the point $\mathbf{Q}$ (corresponding to the chameleon dominated solution) is a stable attractor solution.

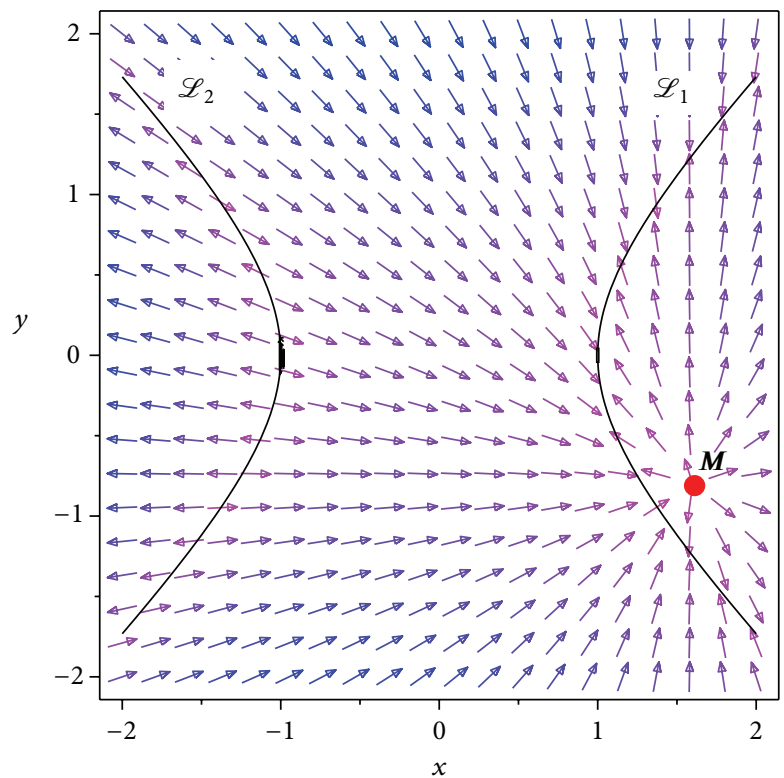

FIGURE 6: The phase space trajectories of the model with $m=1.6$, $n=0.8, \beta=1$, and $\omega=1 / 3$. Like Figure 3 , this figure is plotted also for the case with $u=0$. With these values of parameters, in contrast to Figure 3 , the critical line $\mathscr{L}_{1}$ is stable attractor, while the critical line $\mathscr{L}_{2}$ remains unstable. Also, in this case, the critical point $\mathbf{M}$ is a repeller.

has the phantom-like behavior and can explain the late time cosmic acceleration of the universe in an observationally viable manner.

\section{Summary}

In the current paper, we have studied a hybrid chameleon model in detail. By choosing a runaway potential and by using

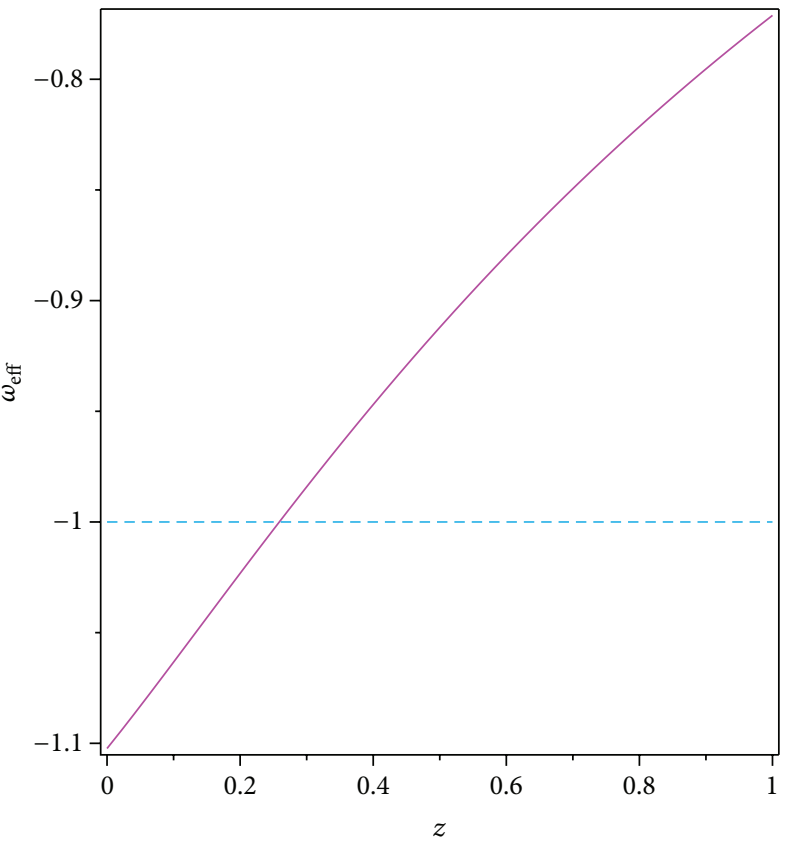

FIGURE 7: The evolution of the effective equation of state parameter of the hybrid chameleon model versus the redshift for the case that $m=1.2, n=0.7, \beta=1$, and $\omega=0$.

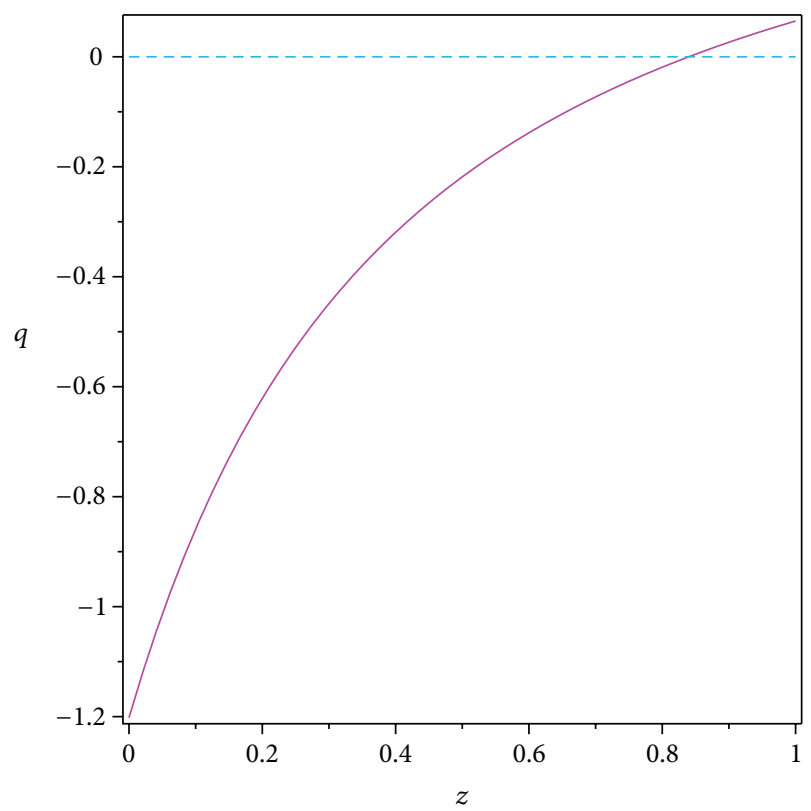

FIGURE 8: The evolution of the deceleration parameter of the hybrid chameleon model versus the red-shift for the case that $m=1.2, n=$ $0.7, \beta=1$, and $\omega=0$.

the coupling term $e^{\kappa \beta(\phi+\varphi)}$, we have introduced an effective chameleon potential. In contrast with runaway potential and coupling term, this effective chameleon potential has a minimum. We have derived this minimum and the masses of the hybrid chameleon field in the minimum. We have found that the minimum of the effective chameleon potential 
is a line and in order for this minimum to exist, there is a constraint on the parameters space of the model. It has been shown that the chameleon field and the matter energy density are related to each other. Also, we have shown that the larger values of the density lead to larger values of the chameleon field's masses. Then we have considered the cosmological dynamics of the hybrid chameleon model. In a dynamical system approach, we have studied the phase space trajectories of the model and its stability. We have found four critical points and two critical lines in our setup and have derived their eigenvalues in order to find the stable solutions. The critical lines and points can be attractor, repeller, and saddle, depending on the values of the parameter spaces. By analyzing two- and three-dimensional phase spaces, it was shown that there are matter domination, scalar field's kinetic term domination, and chameleon domination stable solutions, depending on the values of the parameters space. With some values of the parameters space, we have found the solution where the universe after passing the scalar field's kinetic term dominated era (repeller) and the potential energy dominated and matter dominated era (saddle) reaches a stable attractor chameleon dominated era and remains there forever (one can see the saddle point and attractor in Figure 5). Also, the effective equation of state parameter in this case is below -1 . By adopting the parameter's values used in Figure 5, we have analyzed the late time dynamics of the hybrid chameleon model. We have shown that the universe in this setup has crossed the phantom divide line at $z \simeq 0.26$ and has entered the accelerating phase at $z \simeq 0.84$. So a hybrid chameleon model can explain the late time cosmic acceleration and realize the phantom behavior.

\section{Acknowledgments}

This work has been supported financially by Research Institute for Astronomy and Astrophysics of Maragha (RIAAM) under research project no. 1/2782-34.

\section{References}

[1] A. G. Riess, P. Challis, A. Clocchiatti et al., "Observational evidence from supernovae for an accelerating universe and a cosmological constant," Astronomical Journal, vol. 116, no. 3, pp. 1009-1038, 1998.

[2] S. Perlmutter, G. Aldering, P. G. Castro et al., "Measurements of $\Omega$ and $\Lambda$ from 42 high-redshift Supernovae," Astrophysical Journal Letters, vol. 517, no. 2, pp. 565-586, 1999.

[3] A. G. Riess, L.-G. Sirolger, J. Tonry et al., “Type Ia supernova discoveries at $z>1$ from the hubble space telescope: evidence for past deceleration and constraints on dark energy evolution," Astrophysical Journal Letters, vol. 607, no. 2, pp. 665-687, 2004.

[4] P. Astier, J. Guy, N. Regnault et al., "The supernova legacy survey: measurement of $\Omega_{M}, \Omega_{\Lambda}$ and w from the first year data set," Astronomy and Astrophysics, vol. 447, no. 1, pp. 31-48, 2006.

[5] W. M. Wood-Vasey, G. Miknaitis, C. W. Stubbs et al., "Observational constraints on the nature of dark energy: first cosmological results from the essence supernova survey," Astrophysical Journal Letters, vol. 666, no. 2, pp. 694-715, 2007.
[6] D. N. Spergel, R. Bean, O. Doré et al., “Three-year Wilkinson Microwave Anisotropy Probe (WMAP) observations: implications for cosmology," Astrophysical Journal, vol. 170, no. 2, pp. 377-408, 2007.

[7] G. Hinshaw, M. R. Nolta, C. L. Bennett et al., "Three-year Wilkinson Microwave Anisotropy Probe (WMAP) observations: temperature analysis," Astrophysical Journal, vol. 170, no. 2, p. 288, 2007.

[8] M. Colless, G. Dalton, S. Maddox et al., "The 2dF galaxy redshift survey: spectra and redshifts," Monthly Notices of the Royal Astronomical Society, vol. 328, no. 4, pp. 1039-1063, 2001.

[9] M. Tegmark, M. A. Strauss, M. R. Blanton et al., "Cosmological parameters from SDSS and WMAP," Physical Review D, vol. 69, no. 10, Article ID 103501, 2004.

[10] S. Cole, W. J. Percival, J. A. Peacock et al., "The 2dF Galaxy Redshift Survey: power-spectrum analysis of the final data set and cosmological implications," Monthly Notices of the Royal Astronomical Society, vol. 362, no. 2, pp. 505-534, 2005.

[11] V. Springel, C. S. Frenk, and S. M. D. White, "Review article the large-scale structure of the Universe," Nature, vol. 440, pp. 1137$1144,2006$.

[12] S. P. Boughn and R. G. Crittenden, "A correlation between the cosmic microwave background and large-scale structure in the Universe," Nature, vol. 427, pp. 45-47, 2004.

[13] J. D. McEwen, P. Vielva, M. P. Hobson, E. Martinez-Gonzalez, and A. N. Lasenby, "Detection of the integrated Sachs-Wolfe effect and corresponding dark energy constraints made with directional spherical wavelets," Monthly Notices of the Royal Astronomical Society, vol. 376, no. 3, pp. 1211-1226, 2007.

[14] E. Komatsu, J. Dunkley, M. R. Nolta et al., "Five-year Wilkinson microwave anisotropy probe observations: cosmological interpretation," The Astrophysical Journal, vol. 180, supplement 2, p. 330, 2009.

[15] E. J. Copeland, M. Sami, and S. Tsujikawa, "Dynamics of dark energy," International Journal of Modern Physics D, vol. 15, no. 11, p. 1753, 2006.

[16] S. Nojiri and S. D. Odintsov, "Final state and thermodynamics of a dark energy universe," Physical Review D, vol. 70, Article ID 103522, 15 pages, 2004.

[17] R. R. Caldwell, "A phantom menace? Cosmological consequences of a dark energy component with super-negative equation of state," Physics Letters B, vol. 545, no. 1-2, pp. 23-29, 2002.

[18] N. Arkani-Hamed, P. Creminelli, S. Mukohyama, and M. Zaldarriaga, "Ghost inflation," Journal of Cosmology and Astroparticle Physics, vol. 0404, p. 001, 2004.

[19] F. Piazza and S. Tsujikawa, "Dilatonic ghost condensate as dark energy," Journal of Cosmology and Astroparticle Physics, vol. 2004, p. 0407, 2004.

[20] H. Wei and R. G. Cai, "Interacting vectorlike dark energy, the first and second cosmological coincidence problems," Physical Review D, vol. 73, no. 8, Article ID 083002, 10 pages, 2006.

[21] A. Vikman, "Can dark energy evolve to the phantom?" Physical Review D, vol. 71, no. 2, Article ID 023515, 14 pages, 2005.

[22] A. Anisimov, E. Babichev, and A. Vikman, "The classical stability of the ghost condensate," Journal of Cosmology and Astroparticle Physics, vol. 0506, p. 006, 2005.

[23] B. Wang, Y. G. Gong, and E. Abdalla, "Transition of the dark energy equation of state in an interacting holographic dark energy model," Physics Letters B, vol. 624, no. 3-4, pp. 141-146, 2005.

[24] S. Nojiri, S. D. Odintsov, and S. Tsujikawa, "Properties of singularities in the (phantom) dark energy universe," Physical Review $D$, vol. 71, no. 6, Article ID 063004, 16 pages, 2005. 
[25] S. Nojiri and S. D. Odintsov, "Unifying phantom inflation with late-time acceleration: scalar phantom-non-phantom transition model and generalized holographic dark energy," General Relativity and Gravitation, vol. 38, no. 8, pp. 1285-1304, 2006.

[26] E. Elizalde, S. Nojiri, S. D. Odintsov, and P. Wang, "Dark energy: vacuum fluctuations, the effective phantom phase, and holography," Physical Review D, vol. 71, no. 10, Article ID 103504, 8 pages, 2005.

[27] W. Zhao and Y. Zhang, "Quintom models with an equation of state crossing-1," Physical Review D, vol. 73, no. 12, Article ID 123509, 12 pages, 2006.

[28] P. S. Apostolopoulos and N. Tetradis, "Late acceleration and $w=$ -1 crossing in induced gravity," Physical Review D, vol. 74, no. 6, Article ID 064021, 8 pages, 2006.

[29] U. Alam, V. Sahni, and A. A. Starobinsky, "The case for dynamical dark energy revisited," Journal of Cosmology and Astroparticle Physics, vol. 06, p. 008, 2004.

[30] S. Nesseris and L. Perivolaropoulos, "Comparison of the legacy and gold type Ia supernovae dataset constraints on dark energy models," Physical Review D, vol. 72, no. 12, Article ID 123519, 8 pages, 2005.

[31] M. Libanov, E. Papantonopoulos, V. Rubakov, M. Sami, and S. Tsujikawa, "Ultraviolet stable, Lorentz-violating dark energy with transient phantom era," Journal of Cosmology and Astroparticle Physics, vol. 0708, p. 010, 2007.

[32] R. J. Scherrer and A. A. Sen, "Phantom dark energy models with a nearly flat potential," Physical Review D, vol. 78, no. 6, Article ID 067303, 4 pages, 2008.

[33] F. Briscese, E. Elizalde, S. Nojiri, and S. D. Odintsov, "Phantom scalar dark energy as modified gravity: understanding the origin of the Big Rip singularity," Physics Letters B, vol. 646, no. 2-3, pp. 105-111, 2007.

[34] M. Sami, "Dark energy and possible alternatives," High Energy Physics, http://arxiv.org/abs/0901.0756.

[35] G. Caldera-Cabral, R. Maartens, and L. A. Urena-Lopez, "Dynamics of interacting dark energy," Physical Review D, vol. 79, no. 6, Article ID 063518, 11 pages, 2009.

[36] V. Sahni, "Dark matter and dark energy," Lecture Notes in Physics, vol. 653, pp. 141-180, 2004.

[37] V. Sahni and A. Starobinsky, "Reconstructing dark energy," International Journal of Modern Physics D, vol. 15, p. 2105, 2006.

[38] T. Padmanabhan, "Cosmological constant-the weight of the vacuum," Physics Reports, vol. 380, no. 5-6, pp. 235-320, 2003.

[39] S. M. Carroll, "The cosmological constant," Living Reviews in Relativity, vol. 4, p. 1, 2001.

[40] V. Sahni and A. Starobinsky, "The case for a positive cosmological Lambda-term," Published in the International Journal of Modern Physics D, vol. 9, no. 4, pp. 373-443, 2000.

[41] P. J. E. Peebles and B. Ratra, "The cosmological constant and dark energy," Reviews of Modern Physics, vol. 75, no. 2, pp. 559606, 2003.

[42] E. Bianchi and C. Rovelli, "Why all these prejudices against a constant? ” http://arxiv.org/abs/1002.3966.

[43] R. R. Caldwell, R. Dave, and P. J. Steinhardt, "Cosmological imprint of an energy component with general equation of state," Physical Review Letters, vol. 80, no. 8, pp. 1582-1585, 1998.

[44] I. Zlatev, L. Wang, and P. J. Steinhardt, "Quintessence, cosmic coincidence, and the cosmological constant," Physical Review Letters, vol. 82, no. 5, pp. 896-899, 1999.

[45] R. R. Caldwell, M. Kamionkowski, and N. N. Weinberg, "Phantom energy: dark energy with $w<-1$ causes a cosmic doomsday," Physical Review Letters, vol. 91, no. 7, Article ID 071301, 4 pages, 2003.
[46] A. Sen, "Tachyon matter," Journal of High Energy Physics, vol. 0207, p. 065, 2002.

[47] A. Sen, "Field theory of tachyon matter," Modern Physics Letters A, vol. 17, no. 27, pp. 1797-1804, 2002.

[48] T. Damour, G. W. Gibbons, and C. Gundlach, "Dark matter, time-varying G, and a dilaton field," Physical Review Letters, vol. 64, no. 2, pp. 123-126, 1990.

[49] S. M. Carroll, W. H. Press, and E. L. Turner, "The cosmological constant," Annual Review of Astronomy and Astrophysics, vol. 30, pp. 499-542, 1992.

[50] S. M. Carroll, "Quintessence and the rest of the world: suppressing long-range interactions," Physical Review Letters, vol. 81, no. 15, pp. 3067-3070, 1998.

[51] T. Biswas, R. Brandenberger, A. Mazumdar, and T. Mul- tamaki, "Current acceleration from the dilaton and stringy cold dark matter," Physical Review D, vol. 74, no. 6, p. 063501, 2006.

[52] G. F. Chew and S. C. Frautschi, "Principle of equivalence for all strongly interacting particles within the S-Matrix Framework," Physical Review Letters, vol. 7, no. 10, pp. 394-397, 1961.

[53] T. Damour, F. Piazza, and G. Veneziano, "Violations of the equivalence principle in a dilaton-runaway scenario," Physical Review D, vol. 66, no. 4, p. 046007, 2002.

[54] J.-P. Uzan, "The fundamental constants and their variation: observational and theoretical status," Reviews of Modern Physics, vol. 75, no. 2, pp. 403-455, 2003.

[55] B. Bertotti, L. Iess, and P. Tortora, "A test of general relativity using radio links with the Cassini spacecraft," Nature, vol. 425, no. 6956, pp. 374-376, 2003.

[56] J. Khoury and A. Weltman, "Chameleon fields: awaiting surprises for tests of gravity in space," Physical Review Letters, vol. 93, no. 17, p. 171104, 2004.

[57] D. F. Mota and J. D. Barrow, "Varying alpha in a more realistic universe," Physics Letters B, vol. 581, no. 3-4, pp. 141-146, 2004.

[58] Z. K. Guo, Y. S. Piao, X. M. Zhang, and Y. Z. Zhang, "Cosmological evolution of a quintom model of dark energy," Physics Letters B, vol. 608, no. 3-4, pp. 177-182, 2005.

[59] X. F. Zhang, H. Li, Y. S. Piao, and X. M. Zhang, "Two-field models of dark energy with equation of state across-1," Modern Physics Letters A, vol. 21, no. 3, p. 231, 2006.

[60] R. Lazkoz and G. Leon, "Quintom cosmologies admitting either tracking or phantom attractors," Physics Letters B, vol. 638, no. 4, pp. 303-309, 2006.

[61] R. Lazkoz, G. Leon, and I. Quiros, "Quintom cosmologies with arbitrary potentials," Physics Letters B, vol. 649, no. 2-3, pp. 103$110,2007$.

[62] B. Feng, X. L. Wang, and X. M. Zhang, "Dark energy constraints from the cosmic age and supernova," Physics Letters B, vol. 607, no. 1-2, pp. 35-41, 2005.

[63] B. Feng, M. Li, Y. S. Piao, and X. Zhang, "Oscillating quintom and the recurrent universe," Physics Letters B, vol. 634, no. 2-3, pp. 101-105, 2006. 

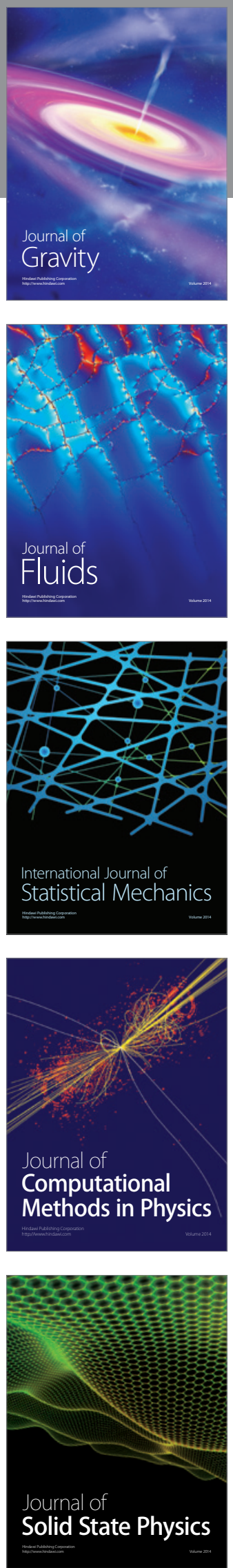

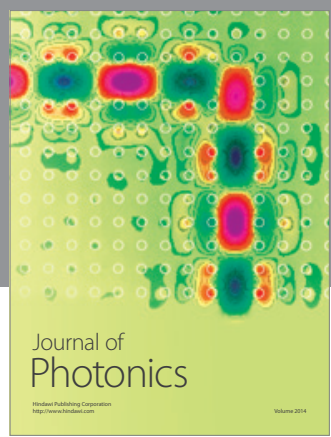

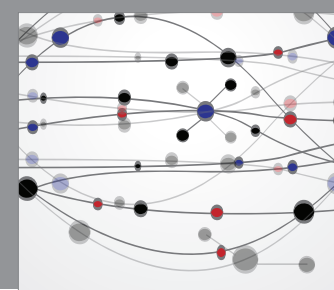

The Scientific World Journal

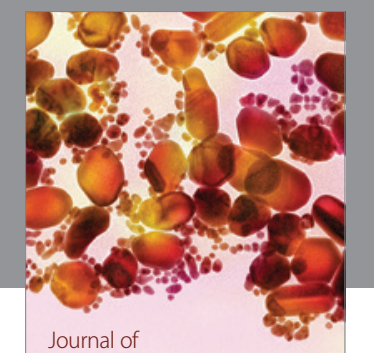

Soft Matter
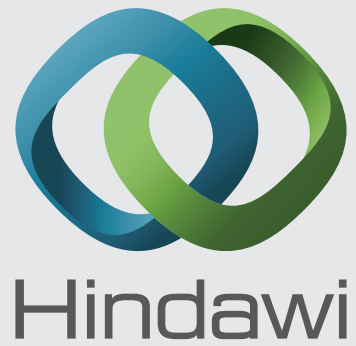

Submit your manuscripts at

http://www.hindawi.com
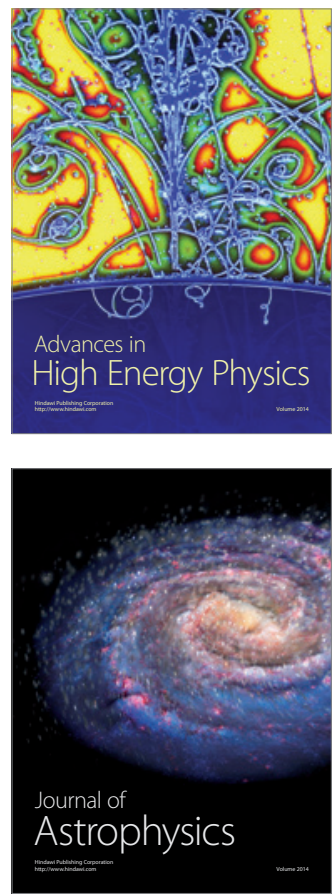
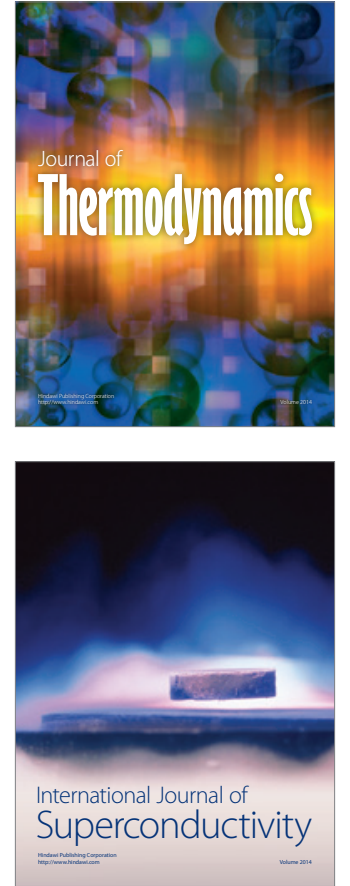
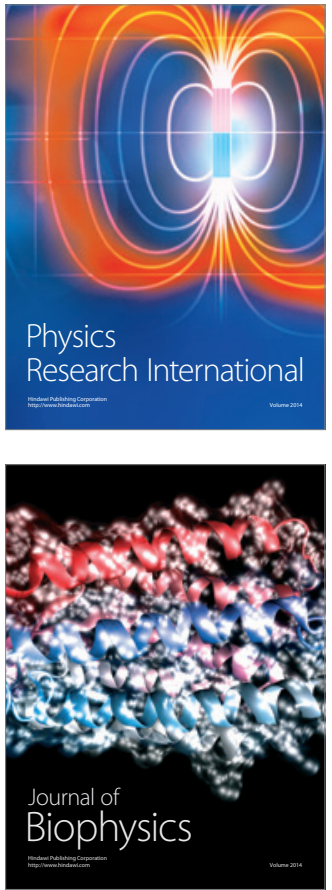
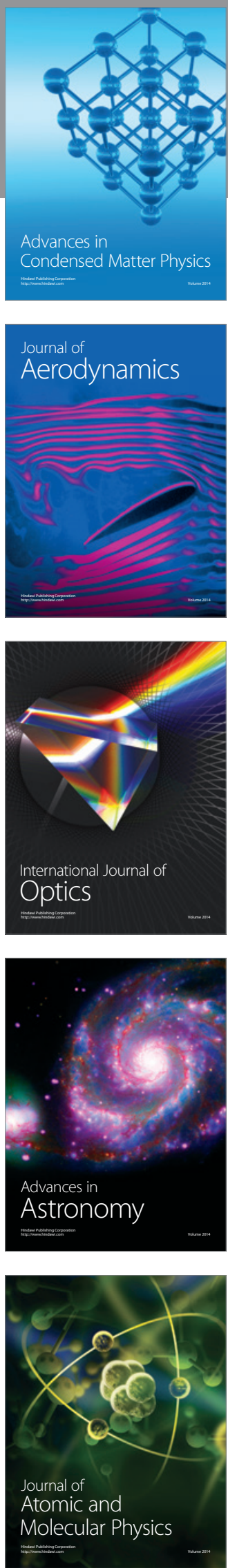